'Facultad de Medicina. Universidad de Chile, Unidad de Neurología, Hospital San Juan de Dios. Santiago. Chile.

${ }^{2}$ Servicio de Urgencia Hospital San Juan de Dios. Santiago. Chile. 3Unidad de Hematología, Hospital San Juan de Dios. Santiago. Chile.

${ }^{4}$ Unidad de Cardiología, Hospital San Juan de Dios. Santiago. Chile. aPhD.

bInterna de $7^{\circ}$ año de Medicina. Universidad de Chile. Santiago. Chile.

'Estudiante de $5^{\circ}$ año de Medicina. Universidad de Chile. Santiago. Chile.

Recibido el 7 de marzo de 2015, aceptado el 28 de enero de 2016.

Correspondencia a: Dr. Carlos Guevara O. neurocrs@hotmail.com

\section{Trombolisis intravenosa en accidente cerebro vascular isquémico agudo en un hospital público de Chile: Análisis prospectivo de 54 casos}

\author{
CARLOS GUEVARA O. ${ }^{1, \mathrm{a}}$, KATERYNA BULATOVA ${ }^{\mathrm{b}}$, FELIPE ARAVENA ${ }^{\mathrm{c}}$, \\ SHEILA CABA $^{2}$, JUAN MONSALVE ${ }^{2}$, HUGO LARA $^{2}$, ELENA NIETO $^{3}$, \\ ISABEL NAVARRETE ${ }^{2}$, MARCELO MORALES ${ }^{4}$
}

\section{Intravenous thrombolysis for ischemic stroke. Experience in 54 patients}

\begin{abstract}
Background: Intravenous thrombolysis with recombinant tissue plasminogen activator (rt-PA) reduces disability in patients with ischemic stroke. However, its implementation in Chilean public general hospitals has been slow and faces some difficulties. Aim: To analyze the results of an intravenous thrombolysis protocol implementation in a public general hospital. Material and Methods: During a lapse of 28 months a standardized protocol for intravenous thrombolysis implemented in the emergency room of a public hospital, was prospectively evaluated. Fifty four patients with ischemic stroke were treated and assessed three months later as outpatients. Results: At three months of follow-up, 66.4\% of patients subjected to thrombolysis had a favorable evolution, defined as having 0 to 1 points in the modified Rankin scale. Intracerebral hemorrhage rate was $11.1 \%$, including $5.5 \%$ of symptomatic intracerebral hemorrhage. Four percent of patients had systemic bleeding complications after thrombolysis. The mortality rate was $14.8 \%$. Conclusions: The success rates, mortality, and complications rate were comparable to the results obtained in international studies, despite of the absence of a stroke unit to manage stroke and its complications.
\end{abstract}

(Rev Med Chile 2016; 144: 442-450)

Key words: Stroke; Thrombolytic Therapy; Tissue Plasminogen Activator.

\section{L} os accidentes cerebrovasculares (ACV) son la primera causa de mortalidad en la población chilena desde el año $2008^{1}$, siendo más frecuente los ACV isquémicos ${ }^{2}$.

Desde 1996, en los países desarrollados, el estándar de manejo del ACV isquémico incluye trombolisis intravenosa (i.v.) con activador del plasminógeno tisular recombinante $(\mathrm{rt}-\mathrm{PA})^{3}$. Su implementación revolucionó los esquemas terapéuticos para manejo de los ACV isquémicos y se asocia a $30 \%$ de reducción de discapacidad en comparación con los pacientes con ACV isquémicos no trombolisados, a pesar de asociarse a un aumentado riesgo de hemorragias intracerebral y sistémica ${ }^{4,5}$.

En América Latina la trombolisis i.v. está subutilizada $^{6,7}$ y en muchos hospitales de referencia del sector público de Chile su implementación está pendiente ${ }^{8}$. El Hospital San Juan de Dios (HSJD) es un centro asistencial responsable de la atención de 7,6\% de la población chilena y tiene un servicio de urgencia (SU) sobrecargado, que atiende aproximadamente 60.000 personas al año?.

El objetivo de este reporte es describir la experiencia de implementación de protocolo de trombolisis i.v. en 54 pacientes con ACV isqué- 
micos en las condiciones sanitarias del HSJD -en su SU y unidad coronaria- entre agosto de 2012 y noviembre de 2014 y analizar los parámetros de atención y resultados clínicos en distintos subgrupos de pacientes.

\section{Pacientes y Métodos}

Se realizo un análisis prospectivo de la implementación de un protocolo de trombolisis i.v. en el HSJD entre agosto de 2012 y noviembre de 2014 y un seguimiento ambulatorio durante al menos 3 meses posterior al procedimiento. El protocolo se basó en el estudio del National Institute of Neurolo- gical Disorders and Stroke (NINDS, 1995)³. Además se incluyeron las conclusiones del estudio ECASS III que demostró la utilidad de la trombolisis i.v. dentro de las $4,5 \mathrm{~h}$ desde el inicio de los síntomas neurológicos ${ }^{10}$.

En la Tabla 1 se muestran los criterios de inclusión y exclusión utilizados en el protocolo de trombolisis i.v. en el HSJD ${ }^{11,12}$.

La estimación de la severidad del compromiso neurológico se realizó mediante la escala National Institute of Health Stroke Scale (NIHSS) que comprende 42 puntos, donde un aumento de puntaje indica mayor compromiso neurológico. Se incluyeron a los pacientes con puntajes $\geq 5 \mathrm{y} \leq 23$, que presentan mayor beneficio de la trombolisis. Los

\section{Tabla 1. Criterios de exclusión e Inclusión para trombolisis Intravenosa}

\section{Criterios de Inclusión}

Síntomas neurológicos por período inferior a 270 min de evolución, con hora de inicio definida

Déficit neurológico entre 5 y 23 puntos en la escala de NIHSS y por más de 30 min

Edad mayor de 18 años

Tomografía axial de cerebro sin evidencias de hemorragia intracraneal

Ausencia de los criterios de exclusión

\section{Criterios de Exclusión}

- Hora inicio de síntomas desconocida, o mayor a 270 min al inicio de la infusión. Si inician durante el sueño se considera la hora en que paciente fue visto asintomático por última vez

NIHSS $>23$ puntos: déficit neurológico severo $0<5$ puntos: déficit leve

ACVb extenso en los últimos 3 meses

Traumatismo craneal encefálico o cirugía del sistema nervioso central en los últimos 3 meses

IAMc dentro de últimos 21 días, a excepción del que ocurre de la manera concomitante con el ACV ${ }^{b}$

Cirugía mayor o biopsia de órgano no compresible en los últimos 14 días

Antecedentes de hemorragia intracraneana

- Síntomas sugerente de hemorragia subaracnoídea

Malformación arteriovenosa o aneurisma cerebral

Antecedente de hemorragia gastrointestinal o urinaria en los últimos 21 días

Antecedentes de coagulopatía (ej: hemofília)

Punción arterial en sitio no compresible o punción lumbar en los últimos 7 días

$\mathrm{PAS}^{\mathrm{d}}>185 \mathrm{mmHg}$ y $\mathrm{PAD}^{\mathrm{e}}>110 \mathrm{mmHg}$ refractaria a la administración de labetalol y/o nitroprusiato

Signos neurológicos que revierten rápidamente

$\operatorname{INR}^{f}>1,5 ;$ TTPK $^{g}>15$ segundos por sobre el límite superior. Uso de heparina de bajo peso molecular en las últimas $24 \mathrm{~h}$ Recuento plaquetario $<100.000 / \mathrm{mm}$

Glicemia $<50$ o $>400 \mathrm{mg} / \mathrm{dl}$

TAC ${ }^{\mathrm{h}}$ de encéfalo sin contraste con ASPECT $<7$

Otros: antecedentes de várices esofágicos, colitis ulcerosa, diverticulitis o pancreatitis aguda, retinopatía diabética con riesgo de hemorragia, neoplasia con riesgo de hemorragia, trauma agudo (fracturas), enfermedad sistémica o infecciosa grave, embarazo o parto dentro del último mes, evidencia de sangrado, sospecha de embolia séptica o de endocarditis infecciosa.

a. National Institute of Health Stroke Scale; b. Accidente cerebrovascular; c. Infarto agudo de miocardio; d. Presión arterial sistólica; e. Presión arterial diastólica; f. International Normalized Ratio; g. Tiempo de protrombina; h. Tomografía axial computada; i. Alberta Stroke Program Early CT Scan score. 
pacientes con $<5 y>23$ puntos, con compromiso mínimo y muy severo, respectivamente, fueron evaluados caso a caso. Pacientes con un NIHSS mayor a 15 fueron considerados como ACV isquémicos severos.

Se practicó una tomografía axial computada (TAC) de cerebro y se usó la escala Alberta Stroke Program Early CT Scan (ASPECTS) para identificar cambios isquémicos tempranos: borramiento de surcos, áreas de hipodensidad y/o pérdida de diferenciación entre sustancia gris y blanca. Estos cambios se identifican en 10 áreas estándares del cerebro y se resta un punto por área afectada del puntaje máximo de 10 , correspondiente a una TAC de cerebro sin cambios agudos ${ }^{13}$. Los pacientes con ASPECTS $<7$ fueron excluidos debido a mayor riesgo de presentar hemorragias intracraneales en relación a la trombolisis i.v. ${ }^{14,15}$.

La trombolisis i.v. con rt-PA fue administrada en $60 \mathrm{~min}$, en dosis de $0,9 \mathrm{mg} / \mathrm{kg}$. El 10\% se administró en bolo durante 2 min y $90 \%$ durante los 58 min siguientes. La forma de administración se basó en los datos del fabricante del rt-PA y del Instituto de Salud Pública de Chile ${ }^{16}$. En un grupo de pacientes coronarios que desarrolló un ACV isquémico durante coronariografías diagnósticas y que recibieron un bolo de heparina no fraccionada (HNF) de 5.000 UI, se usó protamina para revertir su efecto y fue trombolisado con $0,6 \mathrm{mg} /$ $\mathrm{kg}$ de rt-PA ${ }^{17,18}$.
Los pacientes o sus familiares firmaron un consentimiento informado.

En todos los casos se estimaron los tiempos de atención: el tiempo inicio-aguja, medido desde el inicio del cuadro hasta el inicio de la trombolisis i.v.; tiempo puerta-TAC desde el ingreso al SU hasta la realización del TAC y el tiempo puerta-aguja, tomado desde ingreso al HSJD y el inicio de rt-PA, medidos en minutos.

Los tipos de infartos se evaluaron con TAC de cerebro a las $24 \mathrm{~h}$ posteriores a la trombolisis y se describen según la clasificación de Oxfordshire $\left(\right.$ Tabla 2) ${ }^{19}$. Además, se identificaron hemorragias intracraneales (HIC), clasificándolas según el estudio ECASS- $\mathrm{I}^{20}$ : infarto hemorrágico tipo 1 (IH-1): pequeñas petequias sin efecto de masa; infarto hemorrágico tipo 2 (IH-2): petequias confluentes en área del infarto, sin efecto de masa; hemorragia parenquimatosa tipo 1 (HP-1): hematoma en $\leq 30 \%$ del área del infarto con leve efecto de masa; hemorragia parenquimatosa tipo 2 (HP-2): hematoma en $>30 \%$ del área del infarto con efecto de masa importante; hemorragia parenquimatosa a distancia tipo 1 (HPD-1): hematoma pequeño o mediano sin relación con el infarto y hemorragia parenquimatosa a distancia tipo 2 (HPD-2): hemorragia extensa distante del infarto, confluente y con posible efecto de masa .

Hemorragia intracraneal sintomática (HICs) fue definida como HI-2 visualizada en TAC rea-

\section{Tabla 2. Hallazgos en el TACa de cerebro a la 24 h, Clasificación de Oxfordshire y clasificación TOAST'}

\begin{tabular}{|c|c|c|}
\hline $\begin{array}{l}\text { Categoría } \\
\text { Hallazgos en el TAC a de cerebro a las } 24 \text { h }\end{array}$ & $n$ & $\%$ \\
\hline $\begin{array}{l}\text { Clasificación de Oxfordshire } \\
\text { Sin lesión aguda } \\
\mathrm{LACl}^{\mathrm{b}} \\
\mathrm{PACl}^{\mathrm{c}} \\
\mathrm{TACl}^{\mathrm{d}} \\
\text { Hemorragia intracerebral }\end{array}$ & $\begin{array}{r}23 \\
5 \\
11 \\
9 \\
6\end{array}$ & $\begin{array}{r}42,5 \\
9,2 \\
20,3 \\
16,6 \\
11,1\end{array}$ \\
\hline $\begin{array}{l}\text { Clasificación TOASTe de subtipo de } \text { ACV }^{f} \\
\text { Ateroesclerosis de las arterias grandes (trombótico/embólico) } \\
\text { Cardioembólico (de alto o mediano riesgo) } \\
\text { Oclusión de los vasos de calibre pequeño (lacunar) } \\
\text { ACV de otra etiología determinada }^{f} \text { deV de la etiología no determinada } \\
\text { ACV }^{f}\end{array}$ & $\begin{array}{r}25 \\
8 \\
14 \\
0 \\
7\end{array}$ & $\begin{array}{r}46,3 \\
14,8 \\
25,9 \\
0,0 \\
12,9\end{array}$ \\
\hline
\end{tabular}

a. Tomografía axial computada de cerebro sin contraste; b. Infartos lacunares; c. Infarto parcial de la circulación anterior; d. Infarto total de la circulación anterior; e. Trial of ORG 10172 in acute Stroke Treatment; f. Accidente cerebrovascular isquémico; g. Dos o mas causas o estudio negativo o estudio inconcluso. 
lizada hasta 7 días posterior a la trombolisis i.v., asociado a un deterioro neurológico de 4 o más puntos en la escala NIHSS sobre el nivel del ingreso, siendo la principal causa determinante del deterioro neurológico o muerte ${ }^{10}$.

El seguimiento clínico se realizó durante la hospitalización con la escala NIHSS. La escala de Rankin modificada (mRS) fue usada como medida de discapacidad. Los puntajes $m R S$ varían desde 0 puntos (paciente asintomático), y aumenta según el grado de discapacidad hasta 6 puntos (fallecimiento). mRS se usó para determinar el éxito de la trombolisis i.v., que se definió como mRS de 0 o 1 a los tres meses de evolución, siendo los puntaje entre 2 y 5 considerados como falta de respuesta. Mortalidad asociada a tratamiento o mRS de 6, fue aquella de cualquier causa médica ocurrida dentro de los 3 meses posteriores a la trombolisis ${ }^{21,22}$.

Estudio etiológico del ACV isquémico incluyó, entre otros, ecocardiografía transtorácica y ecodoppler carotideo y vertebral y holter de ritmo. Los ACV isquémicos se clasificaron según su etiología usando la escala TOAST (Tabla 2) ${ }^{23}$.

La tasa anual de trombolisis i.v. del HSJD se estimó para el año calendario 2013.

Los estadísticos fueron obtenidos utilizando el programa para Ciencias Sociales (SPSS, versión 22). Las variables continuas se describen con medidas de tendencia central y dispersión: promedio \pm desviación estándar (DS).

\section{Resultados}

Se registraron 731 ingresos de pacientes por ACV isquémico en el HSJD durante el año 2013 y se realizó trombolisis i.v. a 28 pacientes con ACV isquémico durante el mismo año, resultando una tasa anual de trombolisis de 3,8\%.

La Tabla 3 muestra características poblacionales y co-morbilidad de los pacientes trombolisados, que incluye $52 \%$ de mujeres y edad promedio total de 60,9 \pm 15 años. La co-morbilidad más prevalente fue la hipertensión arterial, encontrada en $62,9 \%$ de los pacientes.

Las características clínicas de los pacientes e indicadores de la atención se presentan en la Tabla 4. La severidad promedio de la condición neurológica inicial fue de un NIHSS de $11 \pm 5$ puntos o compromiso moderado. Un $26 \%$ de los pacientes presentaron condición neurológica severa (NIHSS 15-23), con promedio de 18,3 \pm 2 puntos.
Tabla 3. Características Basales de los pacientes Sometidos a Trombolisis Intravenosa

\begin{tabular}{|c|c|c|}
\hline Características demográficas & $\mathbf{n}$ & $\%$ \\
\hline Edad (años \pm DS) & \multicolumn{2}{|c|}{$60,9 \pm 15,0^{a}$} \\
\hline Género femenino & 28 & 51,9 \\
\hline Independencia previa (mRS b $0-1)$ & 54 & 100,0 \\
\hline \multicolumn{3}{|l|}{ Comorbilidades } \\
\hline Hipertensión arterial & 34 & 62,9 \\
\hline Tabaquismo activo & 23 & 42,6 \\
\hline Dislipidemia & 11 & 20,4 \\
\hline Diabetes mellitus tipo 2 & 10 & 18,5 \\
\hline ACVc isquémico previo & 10 & 18,5 \\
\hline Fibrilación auricular & 8 & 14,8 \\
\hline Cardiopatía coronaria & 8 & 14,8 \\
\hline Obesidad & 8 & 14,8 \\
\hline Insuficiencia cardiaca congestiva & 3 & 5,6 \\
\hline Consumo de pasta base de cocaína & 2 & 3,7 \\
\hline Cáncer cervicouterino & 1 & 1,9 \\
\hline Síndrome de antifosfolipicos & 1 & 1,9 \\
\hline
\end{tabular}

a. Expresado en promedio \pm desviación estandar; b. Rankin Score modificado; c. Accidente cerebrovascular.

Tabla 4. Condición de ingreso de los pacientes con indicación de la trombolisis i.v.

\begin{tabular}{|lcc|}
\hline & & $\%$ \\
\hline Categoría & & \\
PAS $^{a}(\mathrm{mmHg} \pm \mathrm{DS})$ & $156 \pm 15$ & - \\
$\mathrm{PAD}^{\mathrm{b}}(\mathrm{mmHg} \pm \mathrm{DS})$ & $86 \pm 31$ & - \\
Glicemia $(\mathrm{mg} / \mathrm{dl} \pm \mathrm{DS})$ & $139 \pm 70$ & - \\
NIHSSc ingreso $\pm \mathrm{DS}$ & $11,1 \pm 5$ & - \\
NIHSSc ingreso 15-23 (severo) & 14 & 25,9 \\
\hline Características de intervención & & \\
Tiempo inicio-aguja \pm DS (min) & $194,4 \pm 59$ & - \\
Tiempo puerta-TAC \pm DS (min) & $42,7 \pm 27$ & - \\
Tiempo puerta-aguja \pm DS (min) & $87,0 \pm 52$ & - \\
Dosis media de rt-PA \pm DS (mg) & $65,5 \pm 10$ & - \\
Tiempo de inicio de tratamiento & & \\
$<3,0 \mathrm{~h}$ & 21 & 38,8 \\
$\geq 3,0$ a $\leq 3,5 \mathrm{~h}$ & 13 & 24,1 \\
$>3,5$ a $\leq 4,0 \mathrm{~h}$ & 7 & 13 \\
$>$ 4,0 y $<4,5 \mathrm{~h}$ & 13 & 24,1 \\
ASPECTS ingreso $<10$ & 4 & 7,4 \\
Días de hospitalización según mRSg & & \\
mRSde egreso 0-1 & 9 & - \\
mRS de egreso 2-5 & 15 & - \\
\hline
\end{tabular}

a. Presión arterial sistólica; b. Presión arterial diastólica; c. National Institute of Health Stroke Scale ${ }^{3}$ d. Tomografía axial computada; e. Activador de plasminogeno tisular recombinante; f. Alberta Stroke Program Early CT Scan ${ }^{9}$; g. Escala de Rankin modificada. 
El tiempo inicio-aguja alcanzó a 194,4 \pm $59 \mathrm{~min}$, mientras que el tiempo puerta-TAC y tiempo puerta-aguja fue de $42,7 \pm 27$ y $87,0 \pm 52$ min, respectivamente.

La Tabla 2 muestra los tipos de infartos determinados al analizar TAC de cerebros a las $24 \mathrm{~h}$ y los tipos de ACV isquémico según los criterios de TOAST $^{23}$.

El resultado del análisis de condición neurológica de los pacientes trombolisados se encuentra resumido en la Figura 1. Según lo obtenido, un NIHSS $<5$ puntos fue aumentando desde $0 \%$ por protocolo al ingreso a $68,5 \%$ al egreso y a $74,0 \%$ a los 90 días.

En la Figura 2 se puede observar los promedios de la evaluación de mRS de los pacientes trombolisados a los 3 meses. El mRS entre 0 y 1 punto (considerado éxito terapéutico) se observó en $66,6 \%$ de los pacientes y un puntaje entre 2 y 5 se halló en $18,6 \%$.

Se registró una tasa de HIC de $11,1 \%$, incluyendo $5,5 \%$ de pacientes con HICs.

Se registraron 8 pacientes fallecidos ( $\mathrm{mRS}$ de 6), lo que se traduce en una tasa de mortalidad de $14,8 \%$. Estos pacientes presentaron una edad promedio de 70,8 \pm 12 años, versus 58,9 \pm 15 años de los pacientes sobrevivientes. El NIHSS de ingreso en pacientes fallecidos fue mayor que en sobrevivientes: $14,5 \pm 5$ versus $10,5 \pm 5$ puntos, respectivamente.

Las características clínicas e imagenológicas se describen con mayor detalle en la Figura 3. Los dos primeros casos estuvieron asociados a una HP-2 y a un manejo farmacológico inadecuado de la
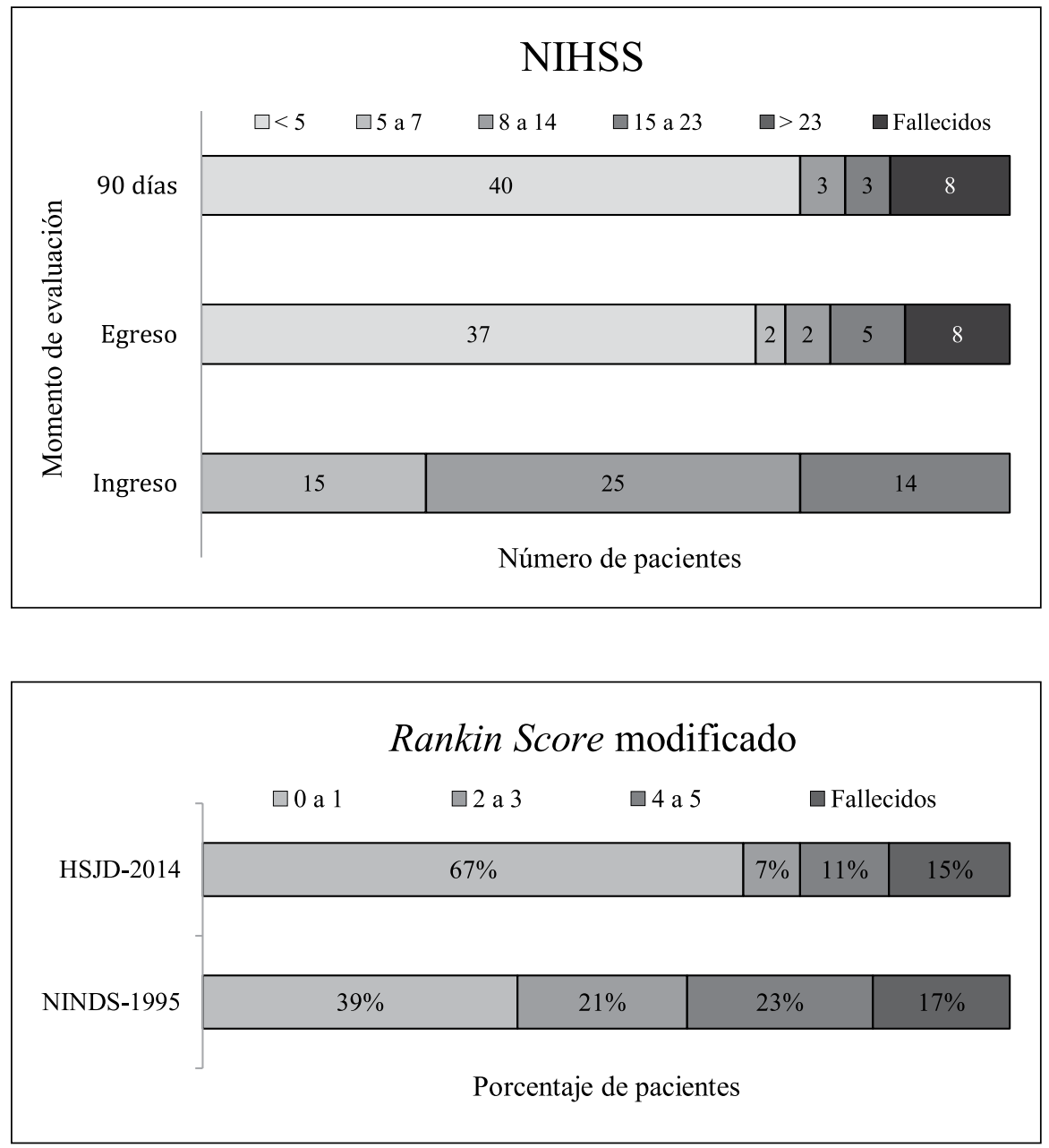

Figura 1. Evolución del NIHSS (National Institute of Health Stroke Scale): severidad de compromiso neurológico entre el ingreso, egreso y control de 90 días posteriores a la trombolisis i.v.

Figura 2. Resultados del control de los pacientes a los 3 meses posteriores a la trombolisis i.v. en pacientes con ACV isquémico. Resultados de puntaje 0-1 en mRS indica una respuesta favorable a la trombolisis. Los valores de suma de los porcentajes pueden no completar $100 \%$ debido al redondeo. 

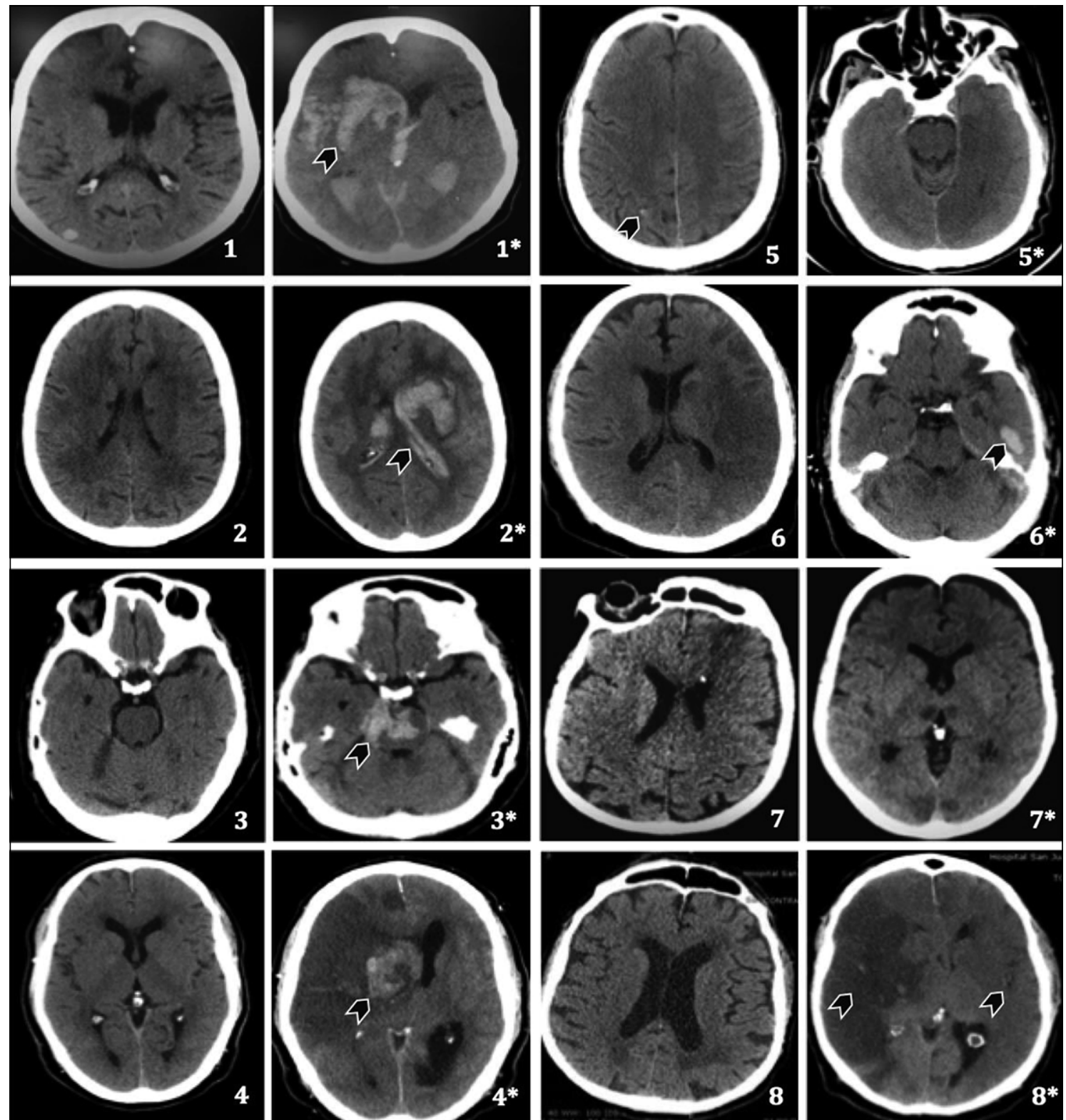

Figura 3. Cortes axiales de las tomografías computadas (TAC) del cerebro sin contraste de los 8 pacientes fallecidos tomadas antes $(1-8)$ y después $\left(1^{*}-8^{*}\right)$ de la trombolisis. Imágenes 5 y $5 *$ corresponden a TACs posteriores al tratamiento.

Caso 1: Mujer de 65 años, hipertensa con PA 191/71 mmHg, asociado a falla en controlar presiones arteriales desarrolla HP-2 $(1 *$, flecha negra).

Caso 2: Paciente de 77 años con presión arterial previa al tratamiento de 235/107 mmHg, mal controlada, desarrolla HP-2 (2*, flecha negra).

Caso 3: Mujer de 79 años desarrolla HP-2 del tronco cerebral $\left(3^{*}\right.$, flecha negra).

Caso 4: Hombre de 72 años con extenso infarto de arteria cerebral media (ACM) y desarrolla HP-1(4*, flecha negra).

Caso 5: Hombre de 64 años. HPD-1. Ambas imágenes de control posterior a la trombolisis. Presenta una pequeña petequia occipital derecha (5, flecha negra), extenso infarto del territorio ACM izquierda $\left(5^{\star}\right)$.

Caso 6: Hombre de 55 años presenta extenso infarto de la ACM izquierda asociada a una HPD-1 a distancia (6*, flecha negra). Caso 7: Hombre de 71 años infartos múltiples occipitales y talámicos, asociados a la neoplasia hepática maligna, desconocida en el momento de decidir la trombolisis.

Caso 8: Infarto extenso bilateral de las ACM $\left(8^{*}\right.$, flecha negra). 
presión arterial, siendo en el primer caso previo al inicio de la trombolisis, y en el segundo a un control hemodinámico inadecuado posterior al procedimiento.

El $18,8 \%$ de las trombolisis se realizaron en pacientes menores de 45 años, con edad promedio de 37,5 \pm 4 años (rango 28 y 42). El NIHSS promedio del ingreso fue de $10,1 \pm 5$ y el de egreso $0,4 \pm$ 0,7 puntos. Ninguno de estos pacientes presentó complicaciones atribuibles a la trombolisis.

Durante el período de estudio se registraron 7 casos de ACV isquémico intrahospitalarios: 4 ocurridos durante una coronariografia diagnóstica y 3 durante hospitalización por otras causas médicas. Los cuatro pacientes con coronariografia presentaron buena evolución neurológica ( $\mathrm{mRS}$ de $0 \mathrm{o}$ 1) y sin hemorragia intracerebral. Dos pacientes con otras patologías $(3,7 \%)$ presentaron complicaciones hemorrágicas sistémicas atribuibles al uso del trombolítico. Una paciente de sexo femenino, con antecedente de irradiación pélvica antigua por cáncer cervicouterino, presentó durante la infusión de rt-PA una hematuria autolimitada, sin repercusión hemodinámica y evolucionó con un mRS de 1. El segundo caso fue en un paciente de sexo femenino, que presentó hemorragia digestiva alta intraprocedimiento, autolimitada, donde estudio posterior reveló doble úlcera gástrica, evolucionando con un mRS de 0 .

El promedio de estadía hospitalaria fue de $10 \pm 5$ días. Cuando los pacientes fueron divididos según puntaje mRS a los tres meses fue de 9,0 \pm 5 días para los pacientes con $0-1$ puntos, $y$ de $15 \pm 10$ días para aquellos con 2-5.

\section{Discusión}

A pesar de que el HSJD no dispone de infraestructura específica para atender a pacientes neurológicos, los valores obtenidos son comparables con los informados en estudios realizados en los países desarrollados. El estudio SITSMOST $(2007)^{24}$ evaluó seguridad y eficacia del tratamiento trombolítico dentro de 3 primeras horas desde el inicio de los síntomas. Este estudio reclutó 6.483 pacientes en centros de países europeos que debían contar con una Unidades de Tratamiento del Accidente Cerebrovascular (UTAC). Se reportó una tasa de éxito (mRS 0-1) de $40 \%$, una tasa de HIC de $17,5 \%$, de HICs de $2,2 \%$ y de mortalidad del $11,3 \%$ a los 3 meses de seguimiento. El estudio ECASS III $(2008)^{10}$ que evaluó la eficacia del tratamiento entre las 3 y 4,5 h de evolución, reclutó 418 pacientes, obteniendo resultados positivos (mRS 0-1 o NIHSS 0-1 o mejora mayor a 8 puntos) en 52\%, una tasa de HIC de $27 \%$, tasa de HICs de $2,4 \%$ y mortalidad de $7,7 \%$. Un reciente meta-análisis de 12 ensayos clínicos randomizados, que incluyó 7.012 pacientes, incluyendo algunas series con ventana terapéutica entre 3 y 6 h, reportó una tasa de HIC de 7,7\%, de HICs fatal durante los primeros 7 días de 3,6\%, y una mortalidad de $19,1 \%{ }^{25}$.

Dados que los estudios de referencia (SITSMOST, ECASS III, NINDS) han considerado un éxito terapeútico a un mRS de $0-1$, hemos usado esta definición, sin embargo, es dable considerar que aquellos pacientes en mRS de 2 a los tres meses pueden considerarse en esta categoría, especialmente si su NIHSS de ingreso fue severo.

En Chile en el 2011 Figueroa et al. reportó en los primeros 19 casos de pacientes trombolisados en un centro hospitalario público, un éxito terapéutico (mRS0-1) de 63\%, mortalidad de $5,2 \%$ y $5,2 \%$ de $\mathrm{HIC}^{8}$. Por otro lado, Lavados et al reportó una mortalidad de $19 \%$ al mes y $28 \%$ a los 6 meses para los ACV isquémicos en ausencia de trombolisis ${ }^{26}$.

Consideramos que la tasa de trombolisis i.v. de 3,8\%, obtenida en este estudio es muy baja; no fue posible cuantificar de forma prospectiva los factores de exclusión. En un estudio canadiense en 24.191 pacientes con isquemia cerebral $11,8 \%$ fue trombolisado, siendo el incumplimiento de la ventana terapeútica la principal causa de exclusión de trombolisis ${ }^{27-29}$.

También destacamos la menor duración en la hospitalización de los pacientes trombolisados (promedio de $10 \pm 5$ días) lo cual hace a esta terapia costo-efectiva en nuestro hospital donde la estadía promedio de pacientes no trombolisados ha sido estimada en 15 días.

La trombolisis i.v. fue segura y efectiva en pacientes jóvenes a juzgar por la ausencia de complicaciones y el éxito terapéutico alcanzado en todos ellos, con un NIHSS promedio de ingreso de $10,1 \pm 5$ y todos en un mRS de 0 o 1 a los tres meses.

Cuatro pacientes recibieron trombolisis i.v a fuera de protocolo debido al uso de HNF en un bolo endovenoso de 5.000 unidades, previo a una angiografía coronaria diagnóstica. 
Enfrentados a la posibilidad de simplemente haber observado a estos pacientes ${ }^{30}$-previamente autovalentes- en la evolución natural de la enfermedad, se consideró oportuno revertir la HNF mediante protamina y con pruebas de coagulación en rango, realizar trombolisis i.v. La protamina es el antídoto del efecto anticoagulante de la HNF, revirtiendo su efecto a los 5 min aproximadamente. La primera pregunta que surge es ¿tendría la protamina efecto procoagulante que empeoraría la clínica de un paciente cursando un ACV isquémico? Existe escasa literatura neurológica al respecto, pero es de uso frecuente en cardiología cuando existen complicaciones hemorrágicas secundarias al uso de HNF, no habiendo evidencia de generar un estado protrombótico ${ }^{31}$. Destacamos que la ausencia de hemorragia intracerebral puedo estar en algún grado asociado además a la dosis menor de trombolítico usada. La frecuencia de reportes y análisis de esta categoria de pacientes ha ido en aumento y requiere mayores definiciones ${ }^{32-34}$.

El tiempo inicio-aguja promedio de 194,4 \pm 59 min fue bastante similar al reportado por Figueroa et al (197,8 min $)^{6}$ en otro hospital público en Chile. La respuesta del equipo sanitario intrahospitalario medida por el tiempo puerta-aguja fue menor en esta serie con 87 min de promedio comparada con la mencionada serie local (111 min).

La mayoría de hospitales públicos en Chile carece de UTAC, a pesar de ser una recomendación tipo A en el manejo de ACV ${ }^{35}$. Dos casos de HICs fatal, estuvieron asociados, aunque no necesariamente causados, a falla en el manejo de la presión arterial. Los cuidados de postrombolisis se realizaron de manera poco estandarizada, teniendo en cuenta la ausencia de UTAC. Si bien la UTAC no es requisito para realizar la trombolisis i.v, su presencia puede razonablemente contribuir a una menor morbi-mortalidad en estos pacientes. Sostenemos que la trombolisis i.v. debe realizarse en un área de tratamiento segura y por un equipo preparado en patología cerebrovascular, esto ayudará a alcanzar estándares de seguridad maximizados y mejorar los paramétros de tiempos y secuelas descritos.

\section{Referencias}

1. Ministerio de Salud de Chile. Indicadores básicos de salud y diez primeras causas de muerte. Chile 2000 a 2010. Departamento de estadísticas e información de salud. http://www.deis.cl
2. Ministerio de Salud de Chile. Informe final estudio de Carga de Enfermedad y Carga Atribuible 2007. http:// www.deis.cl [Consultado el 15 de junio de 2012].

3. National Institute of Neurological Disorders and Stroke rt-PA Stroke Study Group. Tissue plasminogen activator for acute ischemic stroke. N Engl J Med 1995; 333: 15817.

4. Graham GD. Tissue plasminogen activator for acute ischemic stroke in clinical practice: a meta-analysis of safety data. Stroke 2003; 34: 2847-50.

5. Ministerio de Salud de Chile. Guía clínica AUGE. Accidente Cerebrovascular Isquemico en personas de 15 años y más. Serie de las guías clínicas de MINSAL, 2013,http://web.minsal.cl/portal/url/item/722275 4637e58646e04001011f014e64.pdf

6. Avezum A, Costa-Filho FF, Pieri A, Martins SO, Marin-Neto JA. Stroke in Latin America: Burden of Disease and Opportunities for Prevention. Global heart. 2015.

7. Durai Pandian J, Padma V, Vijaya P, Sylaja PN, Murthy JM. Stroke and thrombolysis in developing countries. International journal of stroke: official journal of the International Stroke Society 2007; 2 (1): 17-26.

8. Figueroa-Reyes T, Sáez MD, Mansilla LE, Sánchez VR, Nogales-Gaete J, Delgado BI. Experiencia de trombolisis sistematizada en infarto cerebral agudo en un hospital público de Chile. Rev Med Chile 2011; 139: 1118-27.

9. Ministerio de Salud de Chile. Estadísticas de Atención de Urgencia. Chile, Hospital San Juan de Dios, 2013. Departamento de estadísticas e información de salud. http://www.deis.cl

10. Hacke W, Kaste M, Bluhm E, Brozman M, Dávalos A, Guidetti D, et al. Thrombolysis with Alteplase 3 to 4.5 Hours after Acute Ischemic Stroke. New England Journal of Medicine 2008; 359 (13): 1317-29.

11. Del Zoppo GJ, Saver JL, Jauch EC, et al. Expansion of the time window for treatment of acute ischemic stroke with intravenous tissue plasminogen activator: a science advisory from the American Heart Association/American Stroke Association. Stroke 2009; 40: 2945.

12. Costello CA, Campbell BCV, de la Ossa NP, Zheng TH, Sherwin JC, Weir L. Age over 80 years is not associated with increased hemorrhagic transformation after stroke thrombolysis. Journal of Clinical Neuroscience 2012; 19 (3): 360-3.

13. Pexman JHW, Barber PA, Hill MD, Sevick RJ, Demchuk AM, Hudon ME, et al. Use of the Alberta Stroke Program Early CT Score (ASPECTS) for Assessing CT Scans in Patients with Acute Stroke. American Journal of Neuroradiology 2001; 22 (8): 1534-42.

14. Puetz V, Dzialowski I, Hill MD, et al. The Alberta Stroke Program Early CT Score in clinical practice: what have 
we learned? Int J Stroke 2009; 4 (5): 354-64.

15. Dzialowski I, Hill MD, Coutts SB, et al. Extent of early ischemic changes on computed tomography (CT) before thrombolysis, prognostic value of the Alberta Stroke Program Early CT Score in ECASS II. Stroke 2006; 37 (4): 973-8.

16. Instituto de Salud Publica de Chile. Folleto al profesional.Ref.:MT10692/09-http://www.ispch.cl/sites/default/ files/u7/B-936-05.pdf

17. Matute MC, Masjuan J, Egido JA, Fuentes B, Simal P, Díaz-Otero F, et al. Safety and outcomes following thrombolytic treatment in stroke patients who had received prior treatment with anticoagulants. Cerebrovasc Dis 2012; 33 (3): 231-9.

18. Takayanagi S, Ochi T, Hanakita S, Suzuki Y, Maeda K. The safety and effectiveness of low-dose recombinant tissue plasminogen activator $(0.6 \mathrm{mg} / \mathrm{kg})$ therapy for elderly acute ischemic stroke patients ( $\geq 80$ years old) in the pre-endovascular era. Neurol Med Chir (Tokyo) 2014; 54 (6): 435-40. Epub 2014 Mar 27.

19. Bamford J, Sandercock P, Dennis M, Burn J, Warlow C. Classification and natural history of clinically identifiable subtypes of cerebral infarction. Lancet (London, England) 1991; 337 (8756): 1521-6.

20. Fiorelli M, Bastianello S, Von Kummer R, Del Zoppo G, Larrue V, Lesaffre E, et al; for the ECASS I Study Group. Hemorrhagic transformation within 36 hours of a cerebral infarct. Relationship with early clinical deterioration and 3-month outcome in the European Cooperative Acute Stroke Study I (ECASS I) cohort. Stroke 1999; 30: 2280-4.

21. Rankin J. Cerebral vascular accidents in patients over the age of 60, II: prognosis. Scott Med J 1957; 2: 200-15.

22. Banks JL, Marotta CA. Outcomes validity and reliability of the modified Rankin scale: implications for stroke clinical trials: a literature review and synthesis. Stroke 2007; 38 (3): 1091-6.

23. Adams HP, Bendixen BH, Kappelle LJ, Biller J, Love $\mathrm{BB}$, Gordon DL, et al. Classification of subtype of acute ischemic stroke. Definitions for use in a multicenter clinical trial. TOAST. Trial of Org 10172 in Acute Stroke Treatment. Stroke 1993; 24 (1): 35-41.

24. Wahlgren N, Ahmed N, Dávalos A, Ford GA, Grond M, Hacke W, et al. Thrombolysis with alteplase for acute ischaemic stroke in the Safe Implementation of Thrombolysis in Stroke-Monitoring Study (SITS-MOST): an observational study. The Lancet 2007; 369 (9558): 27582.

25. Wardlaw JM, Murray V, Berge E, del Zoppo G, Sander- cock P, Lindley RL, et al. Recombinant tissue plasminogen activator for acute ischaemic stroke: an updated systematic review and meta-analysis. The Lancet 2012; 379 (9834): 2364-72.

26. Lavados PM, Sacks C, Prina L, Escobar A, Tossi C, Araya $\mathrm{F}$, et al. Incidence, 30-day case-fatality rate, and prognosis of stroke in Iquique, Chile: a 2-year community-based prospective study (PISCIS project). The Lancet 365 (9478): 2206-15.

27. Fang J, Kapral MK, Richards J, Robertson A, Stamplecoski M, Silver FL. The Registry of Canadian Stroke Network: an evolving methodology. Acta neurologica Taiwanica 2011; 20 (2): 77-84.

28. Etgen T, Freudenberger T, Schwahn M, Rieder G, Sander D. Multimodal strategy in the successful implementation of a stroke unit in a community hospital. Acta neurologica Scandinavica 2011; 123 (6): 390-5.

29. Krebes S, Ebinger M, Baumann AM, Kellner PA, Rozanski M, Doepp F, et al. Development and validation of a dispatcher identification algorithm for stroke emergencies. Stroke 2012; 43 (3): 776-81.

30. Chong C, Chiang M, Chiu L. Revisiting unfractionated heparin as a contraindication to acute stroke intravenous thrombolysis. Blood coagulation \& fibrinolysis: an international journal in haemostasis and thrombosis. 2011; 22 (3): 240-1.

31. Ducas J, Chan MC, Miller A, Kashour T. Immediate protamine administration and sheath removal following percutaneous coronary intervention: a prospective study of 429 patients. Catheterization and cardiovascular interventions: official journal of the Society for Cardiac Angiography \& Interventions 2002; 56 (2): 196-9.

32. Diener HC, Foerch C, Riess H, Röther J, Schroth G, Weber R. Treatment of acute ischaemic stroke with thrombolysis or thrombectomy in patients receiving anti-thrombotic treatment. Lancet Neurol 2013; 12 (7): 677-88.

33. Brunner F, Tomandl B, Schröter A, Mellinghoff C, Haldenwanger A, Hildebrandt $\mathrm{H}$, et al. Hemorrhagic complications after systemic thrombolysis in acute stroke patients with abnormal baseline coagulation. European Journal of Neurology 2011; 18 (12): 1407-11.

34. Safouris A, Massaut J, Triantafyllou N, Gazagnes MD, Tsivgoulis G. Safety of IV thrombolysis in an acute stroke patient anticoagulated with unfractioned heparin. J Neurol 2013; 260 (11): 2911-2.

35. Lavados PM, Hoppe WA. Unidades de tratamiento del ataque cerebrovascular (UTAC) en Chile. Rev Med Chile 2005; 133: 1271-3. 